\title{
Arbeitszeitwünsche, Arbeitslosigkeit und Arbeitszeitpolitik
}

Die Verkürzung der Arbeitszeit hat in Deutschland aktuell kaum noch Verfechter. Stattdessen wird von vielen Seiten sogar eine unbezahlte Verlängerung der Arbeitszeit gefordert. Dabei wird jedoch übersehen, dass die abhängig Beschäftigten mehrheitlich der Meinung sind, zu viel zu arbeiten. Und dass sie für eine Reduktion sogar Einkommenseinbußen akzeptieren würden. Eine Auswertung auf Basis des Sozio-ökonomischen Panels (SOEP) ergibt, dass die derzeit weitverbreitete Abweichung der gewünschten von der geleisteten Arbeitszeit eine stark negative Wirkung auf die Zufriedenheit der Beschäftigten hat. Kalkulatorisch könnten durch eine bessere Berücksichtigung der Arbeitszeitpräferenzen der Beschäftigten etwa 2,4 Mio. neue Arbeitsplätze geschaffen werden. ${ }^{1}$

\section{Problemstellung}

Eine (unbezahlte) Verlängerung der Arbeitszeit ist heute in Deutschland bei öffentlichen wie privaten Arbeitgebern ein viel versuchtes Mittel, um die Kosten zu senken. Gleichzeitig ist zu konstatieren, dass trotz eines erkennbaren Rückgangs der Arbeitslosigkeit der verbleibende Bestand zu hoch ist. Vor einem Vierteljahrhundert wäre daraus, wie fast selbstverständlich, die Forderung entstanden, Arbeitszeiten zu verringern, um das vorhandene Arbeitsvolumen, das zumindest kurzfristig als beschränkt angesehen wird, umzuverteilen.

Eine solche Position ist zurzeit fast nicht mehr vernehmbar. Wir möchten dennoch einen neuen Argumentationsversuch in diese Richtung unternehmen. Dazu setzen wir bei den direkt geäußerten Wünschen der Individuen an. Denn das ist eine bisher zu wenig beachtete Dimension in einer Diskussion, die sich fast ausschließlich auf andere Eigenschaften des Arbeitsmarktes stützt, so vor allem auf Lohnhöhen und Qualifikationsmerkmale. Die Präferenzen der Marktteilnehmer sollten jedoch in ökonomischen Betrachtungen immer mit an vorderster Stelle stehen.

Nun wäre die Behauptung übertrieben, dass der Aspekt „Arbeitszeitwünsche der Beschäftigten“ zumindest aus der wissenschaftlichen Diskussion vollständig verschwunden sei. In der Literatur werden sie etwa dann noch wahrgenommen, wenn es um physische und psychische Arbeitsbelastungen sowie um die Betreuung von Kindern geht (Mischau/Oechsle 2005; Nach- reiner et al. 2005). Wir aber wollen zeigen, dass die Zusammenhänge zwischen Arbeitszeit, Gesundheit und Familie nicht nur für Teilgruppen der Beschäftigten bestehen bzw. eventuelle Sonderinteressen abbilden, sondern sehr viele Arbeitnehmerinnen und Arbeitnehmer betreffen.

Im Folgenden werden wir zunächst einige Eigenheiten der gegenwärtigen Diskussion ansprechen (Abschnitt 2). Wir berichten dann über die Ergebnisse des Einflusses eines Auseinanderfallens von Arbeitszeitwunsch und -wirklichkeit auf drei Zufriedenheitsdimensionen im Jahr 2004 (Abschnitt 3 und 4.1). Im nächsten Schritt analysieren wir genauer, welche Merkmale bei Personen dafür verantwortlich sind, weniger oder mehr arbeiten zu wollen (Abschnitt 4.2 und 4.3). Drittens ermitteln wir mit einer Hochrechnung den freiwilligen Umverteilungsspielraum abhängig Beschäftigter in Deutschland (Abschnitt 5). Wir schließen im Abschnitt 6 mit einer Überlegung zur institutionellen Umsetzung einer alternativen Arbeitszeitpolitik.

\section{Eigenheiten der gegen- wärtigen Diskussion}

Arbeitszeitveränderung, um Arbeitslosigkeit zu bekämpfen, hat in Deutschlands Arbeitsmarktforschung keinen besonders guten Ruf mehr. Obgleich die Zusammenschau der empirischen Studien zu den Ergebnissen der gewerkschaftlichen Politik in den 1970er und 1980er Jahren zunächst gar nicht so schlechte Ergebnisse zeigt (Seifert 1993), konzentriert sich die aktuelle wis- senschaftliche Debatte nun auf die umgekehrte Fragestellung, ob nicht vielleicht eine Arbeitszeitverlängerung Entlastung auf dem Arbeitsmarkt bringen könnte.

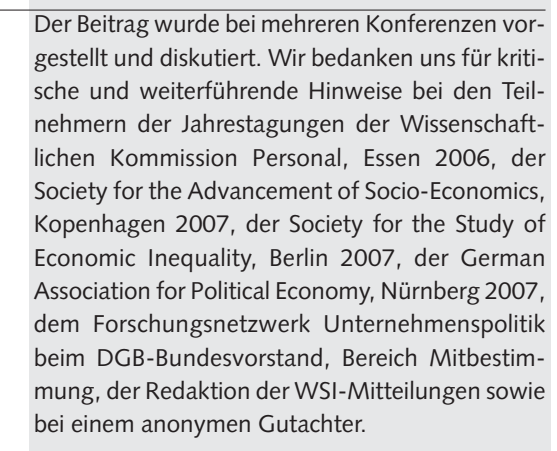
gestellt und diskutiert. Wir bedanken uns für kritische und weiterführende Hinweise bei den Teilnehmern der Jahrestagungen der Wissenschaftlichen Kommission Personal, Essen 2006, der Society for the Advancement of Socio-Economics, Kopenhagen 2007, der Society for the Study of Economic Inequality, Berlin 2007, der German Association for Political Economy, Nürnberg 2007. dem Forschungsnetzwerk Unternehmenspolitik beim DGB-Bundesvorstand, Bereich Mitbestimmung, der Redaktion der WSI-Mitteilungen sowie bei einem anonymen Gutachter.

Gerd Grözinger, Dr., Professor für Bildungs forschung, Collegium Mare Balticum (CMB), Universität Flensburg. Arbeitsschwerpunkte: Bildungsökonomie, Institutionelle Ökonomie, Glücksforschung e-mail: groezing@uni-flensburg.de Wenzel Matiaske, Dr., Professor für allgemeine Betriebswirtschaftslehre, insbesondere Personalwesen und Organisation, Internationales Institut für Management, Universität Flensburg. Arbeitsschwerpunkte: Organisationstheorie, Empirische Organisations- und Personalforschung e-mail: matiaske@uni-flensburg.de Verena Tobsch, Dipl.-Kauffrau, Wissenschaftliche Mitarbeiterin am Internationalen Instititut für Management der Universität Flensburg. Arbeitsschwerpunkte: Führungskräfte- und Genderforschung, Vereinbarkeit von Familie und Beruf, Personalauswahl und -rekrutierung. e-mail:verena.tobsch@uni-flensburg.de 
Die wissenschaftliche Basis für derartige Überlegungen ist praktisch nicht vorhanden. Ein kürzlicher Überblick des Instituts für Arbeitsmarkt- und Berufsforschung (IAB) resümiert, dass zumindest kurzfristig immer mit negativen Beschäftigungseffekten zu rechnen ist (und langfristig alles offen ist, weil von zu vielen Parametern abhängig) (Spitznagel et al. 2005). Dem entspricht auch das Ergebnis einer repräsentativen Umfrage unter Unternehmen im Herbst 2003. Als Antwort auf eine 5 \%-ige Verlängerung der Arbeitszeit ohne Lohnausgleich käme es zu einem Beschäftigungsabbau in etwa gleicher Höhe (Spitznagel/Wanger 2004).

Wenn von einer Arbeitszeitausdehnung so wenig zu erwarten ist, wie wird sie dann begründet? Einer der führenden Arbeitsmarktökonomen Deutschlands plädiert für eine (unbezahlte) Verlängerung der Arbeitszeit, unter anderem mit dem listigen Argument, dass dies ja deswegen zu einer Verringerung des Arbeitsangebots führen könnte, da die gegenwärtige Arbeitszeit vielleicht allzu sehr Präferenzen der Beschäftigten entspräche. Für einen Ökonomen ist das eine merkwürdige theoretische Position: Da soll eine Situation nutzenmäßig bewusst verschlechtert werden, damit sich Marktteilnehmer und Marktteilnehmerinnen zurückziehen. Der dort angeführte empirische Beleg für diese Behauptung ist nicht viel besser: „Immerhin äußerten über $40 \%$ der vollzeitbeschäftigten Männer und Frauen im Rahmen einer Befragung des Sozio-ökonomischen Panels aus dem Jahr 1999, dass die von ihnen geleisteten Stunden mit ihrer präferierten $\mathrm{Ar}$ beitszeit übereinstimmen würden. Allerdings wünschten rund $27 \%$ der vollzeitbeschäftigten Männer beziehungsweise $13 \%$ der Frauen, mehr zu arbeiten, während diese zuletzt genannten Zahlen für Teilzeitbeschäftigte rund $40 \%$ betrugen" (Franz 2004, S. 329).

Dass es auch Erwerbstätige geben könnte, die vielleicht weniger arbeiten möchten, ist für diesen Arbeitsmarktökonomen offensichtlich nicht nachvollziehbar, obwohl der von ihm angeführte Wochenbericht des Deutschen Instituts für Wirtschaftsforschung (DIW) auch solche Zahlen selbstverständlich aufführt und die in der Summe auch schon damals mehrheitlich vorhandene Neigung zu einer Arbeitszeitverkürzung explizit betont. ${ }^{2}$ Was gerade noch an dieser Position stimmt, ist der Verweis auf die hervorzuhebende Rolle des Sozio-ökonomischen Panels (SOEP) für die Frage nach den Präferenzen bei der Arbeitszeit. Es bietet seit Langem die beste Grundlage für eine Analyse der Zeitpräferenzen von Erwerbstätigen. ${ }^{3}$

Das SOEP ist ein von Bund und Ländern im Rahmen der Leibniz Wissenschaftsgemeinschaft finanzierter Paneldatensatz privater Haushalte - jährlich werden also die gleichen Haushalte und die darin lebenden Personen befragt -, der seit mehr als 20 Jahren erhoben und vom DIW bereitgestellt wird. ${ }^{4}$ Ein beeindruckender Umfang von aktuell mehr als 20.000 befragten Personen kommt so als repräsentative Stichprobe der Wohnbevölkerung in Deutschland zusammen. Zwar gibt es für einzelne Gruppen ein Oversampling, z. B. sind Hocheinkommensbezieher, Ausländer und Ostdeutsche überproportional vertreten, jedoch stellt das DIW Hochrechnungsfaktoren bereit, die dieses Oversampling für eine gesamtdeutsche Betrachtung rechnerisch wieder neutralisieren können.

Wir haben die Daten des SOEP aus dem Jahr 2004 ausgewertet (zur Aktualität der Befunde vgl. Abschnitt 4). Es gibt neben dem SOEP noch einige weitere Datensätze zur Untersuchung der Zeitpräferenzen von Erwerbstätigen in Deutschland. Das ISOInstitut zur Erforschung sozialer Chancen hat in den Jahren 1999 und 2003 eigene Befragungen zu diesem Thema unternommen (Bauer et al. 2004), und die Europäische Stiftung zur Verbesserung der Lebensund Arbeitsbedingungen führte 1998 eine europaweite Untersuchung durch, in die auch Deutschland integriert war (Bielinski et al. 2002). Schließlich ist noch der zuletzt 2004 erhobene Ad hoc Labour Market Survey zu nennen, der ebenfalls Fragen nach der wirklichen und der gewünschten Arbeitszeit enthielt (Buscher et al. 2005). Da aber die anderen Studien mit erheblich niedrigeren Fallzahlen arbeiten und sehr viel weniger Variablen aufweisen, bleibt das SOEP die bei Weitem beste Quelle für eine Analyse der gegenwärtigen Situation in Deutschland.

\section{Arbeitszeit und Zufriedenheit im SOEP}

Mehrere im SOEP verwendete Variablen sind für unsere Fragestellung zentral. Erklärt werden sollen verschiedene Dimen- sionen der Zufriedenheit: Arbeitszufriedenheit, Gesundheitszufriedenheit, Lebenszufriedenheit. Die Arbeitszufriedenheit ist eine klassische, d.h. in der empirischen Arbeit wohl bewährte Analysekategorie von Betriebswirten, Psychologen und Soziologen (z. B. Fischer 2006). Die allgemeine Lebenszufriedenheit wird zunehmend als Bewertungsinstrument von Lebensumständen und gesellschaftlichen $\mathrm{Zu}$ ständen nicht nur von Soziologen, sondern verstärkt auch von Ökonomen eingesetzt (Frey/Stutzer 2002; Kahneman et al. 2003; Layard 2005; Böhnke/Kohler 2007). Schließlich hat die Gesundheitszufriedenheit, wie auch die Lebenszufriedenheit, zum einen nicht nur den Vorteil, alle Befragten einzuschließen, sondern zum anderen ist das subjektive Wissen um eine prekäre $\mathrm{Ge}$ sundheit auch ein guter Indikator für objektive Probleme, da Korrelationen mit Größen wie Krankheitstagen, Hospitalaufenthalten, der Sterblichkeit etc. vorliegen (Schwarze et al. 2000).

Im SOEP werden die genannten $\mathrm{Zu}$ friedenheitsaspekte mit diesen einzelnen Fragen erhoben: „Wie zufrieden sind Sie mit Ihrer Gesundheit?“, „Wie zufrieden sind Sie mit Ihrer Arbeit?" (nur für Erwerbstätige), „Wie zufrieden sind Sie gegenwärtig, alles in allem, mit ihrem Leben?". Die Antworten können jeweils auf einer Elfer-Skala gegeben werden, mit 0 als "ganz und gar unzufrieden", mit 10 als "ganz und gar zufrieden“ gekennzeichnet.

Die andere benötigte zentrale Dimension im SOEP betrifft die Arbeitszeit. Zum einen wird dort gefragt: „Wie viel beträgt im Durchschnitt Ihre tatsächliche Arbeitszeit pro Woche einschließlich eventueller Überstunden?" Diese Frage ist der ebenfalls gestellten nach der vereinbarten Wochenarbeitszeit ohne Überstunden vorzuziehen, da in Deutschland zunehmend Überstunden anfallen, teils bezahlt, teils

2 Wörtlich heißt es da etwa: „Die für 1999 auf der Basis des Sozio-oekonomischen Panels (SOEP) aktualisierten Befunde zeigen, dass bei den Beschäftigten weiterhin eine große Bereitschaft zur Verkürzung der Arbeitszeit besteht" (Holst/Schupp 2000, S. 830)

3 Und nicht nur dafür. Wegen der Fülle und der Qualität der hier generierten Informationen ist es eine exzellente Forschungsgrundlage für viele Fragestellungen, die bereits zu mehr als 3.500 wissenschaftlichen Publikationen geführt hat.

4 Für einen aktuellen Überblick siehe etwa Wagner et al. (2006). 
aber auch unbezahlt (Anger 2006). Die zweite wichtige Frage ist zum anderen die nach der Wunscharbeitszeit. Sie wird im SOEP in der für unsere Fragestellung präzisen Form erhoben, nämlich unter Berücksichtigung der damit einhergehenden Kosten, also der Veränderung im Einkommen. Die Frage lautet: „Wenn Sie den Umfang ihrer Arbeitszeit selbst wählen könnten und dabei berücksichtigen, dass sich Ihr Verdienst entsprechend der Arbeitszeit ändern würde; wie viele Stunden in der Woche würden Sie dann am liebsten arbeiten?" Die Antworten werden jeweils in Stunden mit der Möglichkeit einer Kommastelle gegeben.

Diese Informationen sind bisher noch unzureichend ausgewertet worden. Eine Ausnahme stellen die regelmäßigen Analysen des SOEP-Teams am DIW selbst dar, wie sie vor allem von Elke Holst und Jürgen Schupp durchgeführt werden (Holst/ Schupp 1998; Holst/Schupp 2000; Holst 2007). Darüber hinaus hat sich noch Joachim Merz ausführlicher damit beschäftigt (Merz 2002). Typisch für die deutsche Arbeitsmarktdiskussion ist, dass diese Arbeiten praktisch nicht rezipiert werden. ${ }^{5}$

In den SOEP-Auswertungen von Holst und Schupp werden vorwiegend die gewünschten Arbeitszeiten mit der vertraglich vereinbarten Arbeitszeit verglichen. Das ist eine problematische Auswahl des Bezugspunktes, denn dadurch bleiben die (real bedeutenden) Überstunden unberücksichtigt. Entsprechend zu gering erscheint deshalb der Umverteilungsspielraum aufgrund von Präferenzen der Arbeitnehmer.

Anders dagegen die Analyse von Merz. Wie auch bei uns wird hier als Basis die real gearbeitete Zeit genutzt und mit der gewünschten Zeit für die Jahre 1984 und 1995 kontrastiert. Für die Entwicklung Mitte der 1980er bis Mitte der 90er Jahre stellt der Autor fest, dass die Unzufriedenheit mit der eigenen Arbeitszeit stark gewachsen ist, und dass vor allem immer mehr Befragte ihre Arbeitszeit trotz damit einhergehender sinkender Einkommen reduzieren wollen. Dies gilt insbesondere für die abhängig Beschäftigten, während Selbstständige eine höhere Zeitsouveränität realisieren konnten.

\section{Unsere Untersuchung}

Basis für die folgenden Analysen sind die Daten des SOEP aus dem Jahr 2004. Wir haben uns für die vertiefende Analyse dieses Befragungsjahres entschieden, weil hier - über die Standardfragen des SOEP hinaus - für uns interessante Zusatzinformationen erhoben worden sind. Die Daten aus dem Jahr 2004 sind nach wie vor aussagekräftig. Es hat in den Folgejahren lediglich die ungewünschte Mehrarbeit zugenommen (Merz 2002; Anger 2006; Holst 2007), sodass die kritischen Befunde aus dem Jahr 2004 eher eine Untergrenze für die heutige Situation markieren. Untersuchungseinheit sind abhängig Beschäftigte Gesamtdeutschlands, d.h. aktiv erwerbstätige Personen im Alter zwischen 16 und 65 Jahren, die nicht selbstständig oder freiberuflich tätig sind. Eine weitere Abgrenzung erfolgte in Bezug auf unerklärlich hohe Arbeitszeiten, die als Ausreißer interpretiert wurden. D.h. Beschäftigte, die mehr als 80 Stunden pro Woche in der Referenzwoche gearbeitet haben oder mehr als 80 Stunden pro Woche arbeiten wollen, wurden ebenfalls nicht berücksichtigt. Dies betraf einige wenige Fälle.

Zur Berechnung des Einflusses der Arbeitszeit auf die Zufriedenheitsdimensionen muss eine Reihe von miterklärenden Variablen berücksichtigt werden. Aus der Literatur als positiv wirkend sind bekannt: eigenes Arbeitseinkommen als Ausweis beruflicher Anerkennung, das gewichtete Haushaltseinkommen, in fester Beziehung lebend, Bildungsjahre (Matiaske/Mellewigt 2001; Grözinger/Matiaske 2004). Das ProKopf-Haushaltseinkommen ist dabei netto definiert und wurde auf die Mitglieder des Haushalts nach einem Schlüssel verteilt. Benutzt wurde dafür die sogenannte alte OECD-Skala, bei der die erste erwachsene Person (ab 15 Jahre) eine volle Bedarfseinheit erhält, jede weitere jeweils 0,7 , und Personen unter 15 Jahren 0,5. Ein negativer Einfluss auf die Zufriedenheit wird dagegen einer Behinderung (falls vorhanden) unterstellt. Eine ganz besondere Rolle spielt das Alter, und dies nicht nur wegen des hervorzuhebenden quantitativen Einflusses. Es wirkt zusätzlich nicht-linear, zunächst zu-, und dann abnehmend. Deshalb ist es hier zweimal vertreten, einmal als Alter und zusätzlich noch als das Quadrat dieser
Größe. Prognostiziert wird, dass die lineare Größe negativ, die quadrierte aber positiv wirkt.

Neben diesen gut vorhersagbaren Einflüssen gibt es noch eine Reihe von Variablen, bei denen die Wirkungsrichtung aber durchaus offen ist, da Letztere stark kontextabhängig ist. Dennoch sind sie zu kontrollieren, da gelegentlich stärkere Wirkungen festgestellt wurden. Dazu zählen Staatsangehörigkeit (Dummy mit Deutsch $=0$ ), Geschlecht (Dummy mit Mann $=0$ ), Kind im Haushalt (Dummy ohne Kind $=0$ ) sowie auch die Zahl der gearbeiteten Stunden. Wir haben für unsere Analysen zwei weitere Dummyvariablen eingeführt, um die OstWest-Differenz (Dummy mit Westdeutschland $=0)$ und die Hocheinkommensstichprobe (Dummy mit Normalsample $=0$ ) gesondert ausweisen zu können.

Als eigentlich zu untersuchende Größe wird hier die Differenz zwischen der gewünschten und der tatsächlichen Arbeitszeit in Stunden pro Woche eingefügt. Angemerkt sei, dass mit unserer Operationalisierung kein Toleranzbereich für annähernd erreichte Arbeitszeitpräferenzen (wenn z. B. der Unterschied nur eine Stunde pro Woche beträgt) berücksichtigt wird. Dadurch ist es möglich, für die Schätzung des Umverteilungsvolumens eine Obergrenze zu berechnen. Inwieweit Arbeitszeit tatsächlich verteilbar ist, hängt sicherlich aber nicht nur von der individuellen Größe des Arbeitszeitveränderungswunsches $a b$, sondern auch von der Branche, der beruflichen Stellung, der Qualifikation potenzieller und derzeitiger Arbeitnehmer oder den Anforderungen des Arbeitsplatzes selbst.

Da einige Arbeitnehmerinnen und Arbeitnehmer ihre Arbeitszeit ausdehnen, andere sie dagegen einschränken wollen, und es uns im ersten Schritt nur um den Einfluss der Abweichung von Wunsch und Wirklichkeit geht, egal ob konkret Mehroder Minderarbeit gewünscht wird, wurde diese Variable absolut gesetzt. Die zu testende Hypothese lautet entsprechend: Je größer die in Wochenstunden angegebene Arbeitszeitdifferenz zwischen Wunsch und Wirklichkeit ist, desto mehr wirkt das auf eine der Zufriedenheitsdimensionen, und zwar negativ.

\footnotetext{
5 So finden sich auch in der neuesten Ausgabe des führenden deutschen Lehrbuchs zur Arbeitsmarktökonomik zwar Hinweise auf Arbeiten von Holst und Merz, nicht aber auf jene zu den Fragen der Arbeitszeitpräferenzen (Franz 2006)
} 


\section{Tabelle 1: Zufriedenheitsdimensionen (OLS-Regression)}

\begin{tabular}{|c|c|c|c|}
\hline & $\begin{array}{l}\text { Lebens- } \\
\text { zufriedenheit }\end{array}$ & $\begin{array}{l}\text { Gesundheits- } \\
\text { zufriedenheit }\end{array}$ & $\begin{array}{c}\text { Arbeits- } \\
\text { zufriedenheit }\end{array}$ \\
\hline & $\begin{array}{c}\text { Standardisierte } \\
\text { Koeffizienten } \\
\text { Beta }\end{array}$ & $\begin{array}{c}\text { Standardisierte } \\
\text { Koeffizienten } \\
\text { Beta }\end{array}$ & $\begin{array}{c}\text { Standardisierte } \\
\text { Koeffizienten } \\
\text { Beta }\end{array}$ \\
\hline Alter &,$- 762 * * *$ &,$- 612 * * *$ &,$- 509 * * *$ \\
\hline Alter quadriert &, $667 * * *$ &, $406 * * *$ &, $455 * * *$ \\
\hline Grad der Behinderung &,$- 077 * * *$ &,$- 171 * * *$ &,$- 047 * * *$ \\
\hline Dauer der Ausbildung in Jahren &, $068 * * *$ &, $052 * * *$ &, 021 \\
\hline Dummy Person ist weiblich &,- 004 &,- 018 &, 021 \\
\hline Dummy ausländische Staatsbürgerschaft & ,004 &, 016 &,$- 029 * *$ \\
\hline Dummy Ostdeutschland &,$- 112 * * *$ &,$- 050 * * *$ &,$- 052 * * *$ \\
\hline Dummy Hocheinkommensstichprobe &, $057 * * *$ &, $043 * * *$ &, $034 * *$ \\
\hline Dummy Partner im Haushalt &, $087 * * *$ &,- 012 &,- 002 \\
\hline Dummy Kind im Haushalt &, 012 &, 024 &, $061 * * *$ \\
\hline Gewichtetes Haushaltseinkommen &, $064 * * *$ &, $032 *$ &, $054 * * *$ \\
\hline Stundenverdienst &, $078 * * *$ &, $032 *$ &, $056 * * *$ \\
\hline Tatsächliche Wochenarbeitszeit & ,001 &,- 007 &, $039 * *$ \\
\hline Differenz gewünschte und tatsächliche &,$- 087 * * *$ &,$- 052 * * *$ &,$- 125 * * *$ \\
\hline \multicolumn{4}{|l|}{ Arbeitszeit in Wochenstunden } \\
\hline $\mathrm{N}$ & 9.208 & 9.188 & 9.048 \\
\hline $\mathrm{R}^{2}$ (korr.) & 0,075 & 0,095 & 0,038 \\
\hline \multicolumn{4}{|l|}{ Signifikanzniveau: $<0,001(* * *),<0,01\left({ }^{* *}\right),<0,05(*)$} \\
\hline $\begin{array}{l}\text { Quelle: SOEP 2004, alle abhängig beschäftigten Personen } \\
\text { (Berechnungen der Autoren) }\end{array}$ & & & MITTEILUNGEN \\
\hline
\end{tabular}

Dargestellt werden im Folgenden die Ergebnisse einfacher OLS-Regressionen. Zufriedenheit wird zwar zumeist als ordinale Skala interpretiert und damit nur speziellen Verfahren der Regression zugänglich. Aber gerade bei einer feindifferenzierten Antwortvorgabe wie im SOEP führen die beiden Verfahren in der Regel zu ganz ähnlichen Ergebnissen (Grözinger/Matiaske 2004). So auch hier. Wir haben deshalb im Text nur die bekanntere und leichter zu interpretierende OLS-Regression dargestellt.

\subsection{UNERFÜLLTER ARBEITSZEIT- WUNSCH UND WOHLBEFINDEN}

Zunächst einmal ist zu konstatieren, dass die drei Zufriedenheitsdimensionen sich in ihren zentralen Einflussfaktoren sehr ähneln (Tabelle 1). Außerdem wurden alle der oben genannten vermuteten Wirkungsrichtungen bestätigt. Hochsignifikant oder signifikant positiv auf die $\mathrm{Zu}$ friedenheit wirken immer das Quadrat des Alters, zumeist die Ausbildungsjahre, die Höhe des eigenen Stundenverdienstes, die Zugehörigkeit zur Hocheinkommensstichprobe und zumeist noch das Äquivalenzhaushaltseinkommen. Ebenso klar ist das Bild im eindeutig Negativen. Hochsignifikant oder signifikant sind immer die lineare Altersvariable, der Grad einer eventuellen Behinderung und der Dummy für Ostdeutschland.
Daneben gibt es Einflüsse, die nur manchmal auftreten. Bei der Lebenszufriedenheit wirkt das Zusammenleben mit einem Lebenspartner positiv; auch das Vorhandensein von Kindern hat positiven Einfluss auf die Arbeitszufriedenheit. Dieser Befund ist hochsignifikant, während bei den anderen Zufriedenheitsdimensionen der Einfluss der geprüften Variablen unterhalb des Signifikanzniveaus bleibt.

Eine beschränkte Wirkung hat auch die Zahl der gearbeiteten Arbeitsstunden; diese Variable ist nur bei der Arbeitszufriedenheit signifikant positiv. Bei der Arbeitszufriedenheit gibt es eine weitere Besonderheit. Hier ist auch die Variable Staatsangehörigkeit noch signifikant, was bedeutet, dass nicht-deutsche Arbeitnehmer mit ihrer Arbeit unzufriedener sind als ihre deutschen Kollegen.

Die hier den eigentlichen Gegenstand der Untersuchung bildende Variable der Abweichung der gewünschten von der realen Arbeitszeit ist dagegen immer hochsignifikant und bei allen drei Dimensionen mit negativem Einfluss. Damit lautet das erste Fazit: Wer im zeitlichen Umfang anders arbeiten muss als gewünscht, hat eine erhebliche Einbuße an Lebensqualität zu verzeichnen. Wobei diese Negativwirkung bei der Arbeitszufriedenheit relativ am höchsten, bei der Gesundheitszufriedenheit am geringsten ist. Das Ausmaß aber ist in allen drei Dimensionen erheblich. Die Differenz der realen zur gewünschten Ar- beitszeit ist z. B. in der Bedeutung auf die Zufriedenheit etwa so stark wie der Einfluss des verfügbaren Einkommens oder auch der Grad einer gegebenenfalls vorliegenden Behinderung, wie die in Tabelle 1 angegebenen (Beta-)Werte zeigen. ${ }^{6}$

In dieser ersten Berechnung wurde gezeigt, welche Bedeutung ein Auseinanderfallen von Wunsch und Wirklichkeit bei der Arbeitszeit auf zentrale Zufriedenheitsdimensionen hat. Die Richtung der Abweichung und ihre Verankerung bei speziellen Gruppen oder Lebenslagen spielte hier noch keine Rolle. Eine derart differenzierte Analyse ist Gegenstand des nächsten Abschnitts.

\subsection{WER WILL SEINE ARBEITSZEIT WIE VERÄNDERN?}

Als zu erklärende Variablen stehen im Folgenden nicht mehr die Zufriedenheitsdimensionen zur Diskussion, sondern das Ziel ist jetzt, festzustellen, welche Beschäftigtengruppen den Wunsch haben, ihre Arbeitszeit zu verändern. Und da anzunehmen war, dass der Wunsch nach Mehrarbeit von einer ganz anderen Situationsspezifik als das bekundete Interesse nach niedrigerer Arbeitszeit getragen wird, sind die Berechnungen getrennt für beide Richtungen durchgeführt worden. Beschrieben wird also nun, wer einen besonders großen Wunsch nach bezahlter Ausdehnung der Arbeitszeit hat und wer dagegen eine stärkere Reduzierung der Arbeitszeit präferiert (Tabelle 2). Die Gruppe der mit ihrer aktuellen Arbeitszeit voll Zufriedenen spielt demnach bei diesem Analyseschritt keine Rolle mehr.

Die erklärenden Variablen entsprechen den oben angegebenen, jedoch mit zwei kleinen Modifikationen. Es stellte sich heraus, dass jetzt das Alter linear wirkt, und auf den quadratischen Term deshalb hier verzichtet werden konnte. Bei dem Wunsch nach Veränderung der Arbeitszeit war der Grad einer evt. vorliegenden Behinderung nie signifikant, sodass auch diese Variable im Folgenden ausgelassen wurde.

Besonders viel mehr arbeiten möchten die eher Jüngeren, stärker auch die Männer,

\footnotetext{
6 Die Beta-Werte normieren alle Inputwerte der Variablen, sodass deren Dimension keine Rolle spielt und ein unmittelbarer Vergleich untereinander erfolgen kann.
} 
die Erwerbstätigen mit ausländischer Staatsbürgerschaft, die Bewohner Ostdeutschlands, die nicht mit einem Lebenspartner oder Kindern zusammenwohnen, die ein niedriges Haushaltseinkommen, aber einen höheren Stundenlohn haben und die vor allem jetzt wenig arbeiten.

Fast ganz spiegelbildlich sieht die Situation bei denjenigen aus, die sich eine Arbeitszeitreduktion wünschen. Hier sind es die eher Älteren, mehr die Frauen, die in Westdeutschland Lebenden, die mit Lebens- oder Ehepartner zusammenwohnen, die mit höherem Haushaltseinkommen, aber mit niedrigerem Stundenlohn, und die vor allem viel arbeiten. Nur die Staatsbürgerschaft bleibt im Unterschied zur ersten Berechnung ohne messbaren Einfluss. Dafür kommen als ebenfalls positiv hochsignifikant hier noch die Einflüsse der Bildungsjahre und der $\mathrm{Zu}$ gehörigkeit zur Hocheinkommensstichprobe dazu.

Das Zusammenleben mit Kindern im Haushalt verstärkt (hochsignifikant) den Wunsch nach Reduzierung der wöchentlichen Arbeitszeit. Eine andere Untersuchung, ebenfalls auf Basis der Daten des SOEP, kommt zu dem Ergebnis, dass zumindest bei Müttern mit kleinen Kindern Teilzeitarbeit die vielfach präferierte Beschäftigungsform ist (Trzcinski/Holst 2003). ${ }^{7}$ Dieser Befund wird auch hier sichtbar, wenn nämlich die Berechnung noch einmal nach dem Geschlecht differenziert wird: Während die Existenz von Kindern beim Wunsch nach Arbeitszeitreduktion bei Männern keinen Einfluss ausübt, ist er bei den Frauen signifikant positiv.

Wer mehr und wer weniger arbeiten möchte, gehört also ganz unterschiedlichen Gruppen an. In beiden Fällen ist jedoch der bei Weitem stärkste Einfluss bei der aktuellen Arbeitszeit zu finden: Wer mehr arbeiten möchte, arbeitet aktuell wenig, wer dagegen weniger Arbeit anstrebt, arbeitet zurzeit viel. Deshalb ist es zunächst von Interesse, wie groß die durchschnittliche Differenz zwischen gewünschter und tatsächlicher Arbeitzeit für diese offensichtlich unterschiedlichen Lebenssituationen ist. Danach kann noch die Frage beantwortet werden, welche gesamtwirtschaftlichen Folgen es hätte, wenn alle Zeitpräferenzen sich erfüllen würden.

\begin{tabular}{|c|c|c|c|c|}
\hline & \multirow[t]{2}{*}{ Verlängerung } & \multicolumn{3}{|c|}{ Verkürzung } \\
\hline & & Alle & Nur Männer & Nur Frauen \\
\hline Alter &,$- 064 *$ & $117 * * *$ &, $062 * * *$ &, $153 * * *$ \\
\hline Dauer der Ausbildung in Jahren &,- 022 &, $064 * * *$ &, $048 * *$ &, $093 * * *$ \\
\hline Dummy Person ist weiblich &,$- 123 * * *$ &, $210 * * *$ & & \\
\hline Dummy ausländische Staatsbürgerschaft &, $097 * * *$ & ,017 &, $035 *$ & ,014 \\
\hline Dummy Ostdeutschland &, $161 * * *$ &,$- 070 * * *$ &,- 026 &,$- 116 * * *$ \\
\hline Dummy Hocheinkommensstichprobe &,- 046 &, $045 * * *$ &, 022 &, $042 *$ \\
\hline Dummy Partner im Haushalt &,$- 091 * * *$ &, $038 * *$ &,- 002 &, $091 * * *$ \\
\hline Dummy Kind im Haushalt &,$- 058 *$ &, $080 * * *$ &,- 013 & $134 * * *$ \\
\hline Gewichtetes Haushaltseinkommen &,$- 066 *$ &, $066 * * *$ & ,016 &, $065 * *$ \\
\hline Stundenverdienst &, $092 * * *$ &,$- 117 * * *$ &,$- 065 * *$ &,$- 100 * * *$ \\
\hline Tatsächliche Wochenarbeitszeit &,$- 511 * * *$ &, $633 * * *$ &, $686 * * *$ &, $537 * * *$ \\
\hline $\mathrm{N}$ & 1.521 & 5.033 & 2.865 & 2.167 \\
\hline $\mathrm{R}^{2}$ (korr.) & 0,230 & 0,366 & 0,487 & 0,279 \\
\hline \multicolumn{5}{|c|}{ Signifikanzniveau: $<0,001(* * *),<0,01(* *),<0,05(*)$} \\
\hline $\begin{array}{l}\text { Quelle: SOEP 2004, alle abhängig beschäftigten Per } \\
\text { Arbeitszeitveränderung (Berechnungen der Autoren }\end{array}$ & nen mit gewünsc & & M/SI & TEILUNGEN \\
\hline
\end{tabular}

\subsection{DIMENSION UND BEDEUTUNG GEWÜNSCHTER ARBEITSZEITEN}

Während die bisherigen Berechnungen auf der Individualebene stattfanden, müssen nun für die nächsten Kalkulationen $\mathrm{Ge}$ wichtungsfaktoren angewandt werden. Sie sorgen dafür, dass die Ergebnisse als gültig für alle Beschäftigtengruppen angesehen werden können, selbst wenn einzelne Gruppen im Gesamtdatensatz überrepräsentiert sind. ${ }^{8}$ Tabelle 3 weist die hochgerechneten und somit verallgemeinerbaren Befunde aus.

Die Gruppen der Beschäftigten, die ihre Arbeitszeit verlängern wollen, und diejenigen, die sie verkürzen wollen, unterscheiden sich erheblich in ihrer Ausgangslage bezüglich der realen Arbeitszeit. Beide Wünsche sind leicht nachzuvollziehen, arbeiten doch die Ersteren im Schnitt nur knapp 26 Stunden, die anderen aber fast 43 Stunden. Es ist interessant, dass die Wunschzeit der beiden Kategorien fast deckungsgleich ist und bei etwa 34,5 Stunden liegt. Dort befindet sich auch der Wert von denjenigen, die im Moment mit ihrer Arbeitszeit voll zufrieden sind. Trotz aller Spezifik der zweifellos vorhandenen individuellen Lösungswünsche liegt der jeweilige statistische Durchschnittswert der Wunscharbeitszeit aller drei Gruppen doch sehr nahe beieinander.

An dieser Aufteilung zeigt sich klar die Bedeutung nicht-wunschgemäßer Arbeitszeiten. Nur etwas mehr als ein Viertel der Erwerbstätigen ist mit der Ist-Situation zufrieden. Ein knappes Fünftel würde gerne mehr arbeiten, vor allem Männer und Ost- deutsche, die derzeit eine nicht erwünschte Teilzeitstelle innehaben. Eine eindeutige Mehrheit jedoch möchte die tatsächliche Arbeitszeit verkürzen. Und dies im Schnitt um acht Stunden pro Woche, wobei sich diese hohe Zahl als eine Mischung aus unerfüllten Teilzeitwünschen, Rückführung von Überstundenbelastungen oder einfach dem Interesse an etwas mehr Freizeit erklärt.

Werden beide Wunschdimensionen gegeneinander aufgerechnet - der nach Verlängerung und der nach Verkürzung bleibt ein rechnerischer Nettoeffekt übrig, der pro Erwerbstätigen eine Verkürzung um 2,77 Stunden ergibt. Gemessen an der real gearbeiteten Wochenzeit von 37,23 Stunden ist das der Wunsch nach einer mittleren Senkung der Arbeitszeit aller Beschäftigten um etwa 7,5\%. Oder anders ausgedrückt: Wenn die Minderung der Arbeitsleistung durch eine solche Veränderung der Arbeitszeit sich in direkter Weise in neue Arbeitsplätze umsetzen würde, wären über 2,4 Mio. neue Jobs mit durchschnittlicher Arbeitszeit entstanden.

7 Ähnlich ist das Ergebnis eines Gutachtens für das Familienministerium (Rürup/Gruescu 2005). Es gilt für Deutschland (zumindest noch) die Aussage: "Letztendlich wird die von Frauen gewünschte und ausgeübte Erwerbszeit in erheblichem Umfang vom Vorhandensein von Kindern beeinflusst... Für Männer spielen diese Faktoren dagegen keine derartige Rolle" (Holst/Trzcinski 2005, S. 214).

8 Da für die Hocheinkommensbezieher keine Gewichtungsfaktoren vorliegen und ihre quantitative Bedeutung gering ist, bleibt diese Gruppe bei den folgenden Berechnungen unberücksichtigt. 
Tabelle 3 Arbeitszeitwünsche und Umverteilungsvolumen

\begin{tabular}{|c|c|c|c|c|c|c|c|c|}
\hline \multicolumn{3}{|l|}{ 1a) Arbeitszeitpräferenzen } & & & & \multicolumn{3}{|c|}{ 1b) Umverteilungsvolumen } \\
\hline & & & \multicolumn{4}{|c|}{ Durchschnittliche Wochenarbeitszeit in Stunden } & \multicolumn{2}{|c|}{$\begin{array}{l}\text { Rechnerisches Umverteilungsvolumen } \\
\text { für Gesamtdeutschland }\end{array}$} \\
\hline \multirow[t]{2}{*}{$\begin{array}{l}\text { Präferenz für Veränderung } \\
\text { der Wochenarbeitszeit }\end{array}$} & $\begin{array}{l}\text { Anzahl der } \\
\text { Beschäftigten }\end{array}$ & $\ln \%$ & Vereinbart & Tatsächlich & Gewünscht & $\begin{array}{l}\text { Differenz } \\
(5)-(4)\end{array}$ & $\begin{array}{l}\text { In Stunden } \\
\text { pro Woche } \\
(6)^{*}(1)\end{array}$ & $\begin{array}{c}\text { Arbeitsplätze } \\
\text { (bei gew. } \\
\text { Wochenarbeitszeit) } \\
(7) /(5)\end{array}$ \\
\hline & 1 & 2 & 3 & 4 & 5 & 6 & 7 & 8 \\
\hline Verkürzung gewünscht & 16.230 .426 & 53,97 & 37,05 & 42,59 & 34,59 & $-8,00$ & -129.807 .944 & -3.752 .641 \\
\hline keine Veränderung & 8.370 .318 & 27,83 & 33,56 & 34,39 & 34,39 & 0,00 & 0 & 0 \\
\hline Verlängerung gewünscht & 5.475 .012 & 18,20 & 26,34 & 25,68 & 34,15 & 8,48 & 46.405 .207 & 1.358 .698 \\
\hline INSGESAMT & 30.075 .756 & 100,00 & 34,32 & 37,23 & 34,46 & $-2,77$ & -83.402 .736 & -2.420 .537 \\
\hline
\end{tabular}

Quelle: SOEP 2004, alle abhängig Beschäftigten ohne Hocheinkommensstichprobe, gewichtet und hochgerechnet (Berechnungen der Autoren).

WSI MITTEILUNGEN

\section{Vergleichsstudien}

Auch wenn man zugesteht, dass eine Einszu-eins-Umsetzung nicht wahrscheinlich ist - es gibt qualifikatorischen wie regionalen Mismatch, darüber hinaus entstehen Produktivitätsschübe bei Arbeitszeitverkürzungen, - steckt hier doch ein erheblicher Beitrag zur Senkung der nach wie vor zu hohen Arbeitslosigkeit in Deutschland. Der durch eine bessere Umsetzung von individuellen Arbeitszeitpräferenzen gegebene Umverteilungsspielraum wird aber in der öffentlichen Diskussion weder wahrgenommen noch vermutet.

Deshalb ist es nützlich, einen Blick auf die beiden anderen größeren Umfragen zu diesem Thema zu werfen, um das hier vorgestellte Ergebnis damit abzugleichen. 1998 wurden in Europa (EU und Norwegen) insgesamt 30.000 Personen der Wohnbevölkerung im Alter von 16 bis 65 nach ihren faktischen Arbeitszeiten gefragt: „Alles in allem: Wie viele Stunden pro Woche arbeiten Sie derzeit im Durchschnitt?" In ähnlicher Weise wie beim SOEP wurde dabei auch der Wunsch nach Veränderung erhoben. Die diesbezügliche Frage lautete: „Nehmen Sie bitte einmal an, dass Sie (und Ihr Partner) die freie Wahl hätten, was Ihre Arbeitszeit angeht und denken Sie dabei bitte auch an das notwendige Geldverdienen: Wie viele Stunden pro Woche würden Sie selbst derzeit pro Woche arbeiten?" (Bielinski et al. 2002, S. 26).

Auch wenn im Vergleich zum SOEP eine etwas unterschiedliche Frageformulierung vorliegt, was durchaus Auswirkung auf die Angaben der Befragten haben kann (Schief 2003), bestätigen die dortigen Ergebnisse beeindruckend das Gesamtbild unserer Befunde. In Europa insgesamt möchten abhängig Beschäftigte fast $10 \%$ weniger arbeiten und ihre Arbeitszeit von durchschnittlich 37,7 auf 34 Stunden pro Woche reduzieren (Bielinski et al. 2002, S. 67). Und für Deutschland allein ist das ganz ähnlich, im Durchschnitt wird eine Verringerung von 37,5 auf 33,7 Stunden gewünscht (Bielinski et al. 2002, S. 71). ${ }^{9}$

Gleiches gilt für die ISO-Umfrage 2003. Auf die Frage: „Wenn Sie sich aussuchen könnten, wie viele Stunden Sie arbeiten und sich dabei Ihr Einkommen in etwa entsprechend Ihrer Arbeitszeit erhöhen oder vermindern würde: Wie viele Stunden würden Sie dann gerne in der Woche arbeiten?" (Bauer et al. 2004, S. 13, Anhang) wollten Vollzeitbeschäftigte ihre Wochenarbeitszeit um 4,1 Stunden reduzieren, Teilzeitarbeitende diese um 2,5 Stunden erhöhen. Da das Verhältnis der ersten zur zweiten Gruppe nach der hier benutzten Definition etwa Drei zu Eins beträgt (Bauer et al. 2004, S. 53, 62) kommt es zu einem Nettoeffekt von etwa 2,5 Stunden pro Woche.

Das von uns errechnete Umverteilungsvolumen beträgt für 2004 83,4 Mio. Arbeitsstunden. Das korrespondiert mit dem Gutachten von Rürup und Gruescu, die anhand von Daten des Statistischen Bundesamtes für Deutschland im Jahr 2001 ein Umverteilungsvolumen von insgesamt 80,7 Mio. Wochenstunden mit einer durchschnittlich gewünschten Arbeitszeit von 34,8 Stunden pro Woche errechneten (eigene Berechnungen anhand Rürup/ Gruescu 2005, Tabelle S. 17).

Schließlich ist noch auf eine etwas ältere Studie der McKinsey-Unternehmensberatung zu verweisen, die in den 1990er Jahren die Chancen einer stärkeren Teilzeitbeschäftigung in Übereinstimmung mit Arbeitszeitwünschen von Beschäftigten wie
Betriebsinteressen untersuchten. Ihr berücksichtigt man die Autorenschaft - erstaunliches Ergebnis war, dass dadurch etwa 1,4 Mill. Vollzeit- bzw. 1,9 Mill. neue Teilzeitstellen geschaffen werden könnten (McKinsey and Company 1994).

Das zweite Fazit lautet somit: Es gibt in Deutschland ein großes Potenzial an gewünschter Arbeitszeitreduktion, das nicht umgesetzt wird. Zugleich gibt es ein hohes Maß an Arbeitslosigkeit. Es stellt sich die Frage, welche Maßnahmen hier eine bessere Übereinstimmung der Präferenzen sowohl der Beschäftigten wie der Erwerbslosen erbringen könnten.

\section{Politisch-institutionelle Schlussüberlegungen}

Historiker zeigen: Die Sozialgeschichte Deutschlands ist geprägt von einem lang anhaltenden, fast linearen Trend sinkenden Jahresarbeitsvolumens pro Einwohner. Von der ersten Zählung 1882 bis zum letztausgewerteten Jahr 2002 ist dies gut dokumentiert (Schildt 2006). An diesem Trend wird implizit auch deutlich, dass Unternehmen in ihrer Arbeitsorganisation hinreichende Flexibilitätsspielräume haben. So sind z. B. die Betriebslaufzeiten in Deutschland trotz Arbeitszeitverkürzung

9 Bei einer anderen europaweiten Umfrage, die zuletzt 2004 durchgeführt wurde, sind leider die realen und gewünschten Stunden der Erwerbstätigen nur schematisch erhoben worden. Aber auch hier übertrifft in Deutschland (wie Europa insgesamt) der Wunsch nach Arbeitszeitreduktion den nach Verlängerung um ein Mehrfaches (Buscher et al. 2005). 
angestiegen, etwa im Verarbeitenden Gewerbe von 67,9 Stunden im Jahr 1984 auf 71,8 Stunden 1996 (Lehndorff 2001, S. 49).

In den letzten anderthalb Dekaden jedoch ist keine weitere Verkürzung mehr zu beobachten (Bosch et al. 2005). Die Gründe dafür sind vielfältig. Bedeutsam waren sicherlich die hohe Arbeitslosigkeit, stagnierende bis zurückgehende Reallöhne (Bosch et al. 2005) und eine damit einhergehende Schwäche der Gewerkschaftsbewegung, die früher der Garant für eine Politik der Arbeitszeitverkürzung war. Diese Rahmenbedingungen werden sich vermutlich auch in naher Zukunft nicht ändern. Zugleich sind in Deutschland, wie wir zeigen konnten, sowohl ein hohes Maß an individueller Unzufriedenheit mit der Arbeitszeit als auch ein Potenzial zur Arbeitsumverteilung festzustellen.
Damit stellt sich die Frage neu, wie die vielfältigen Präferenzen der Beschäftigten an individuelle Arbeitzeitlösungen angemessen umgesetzt werden und zugleich die Wünsche der Arbeitslosen nach Beschäftigung Berücksichtigung finden können. Diese Differenziertheit in den Vorstellungen von personenbezogenen Arbeitszeiten und das Misstrauen gegenüber Standardlösungen lassen sich nicht nur im SOEP gut nachweisen. Auch die Auswertung einer ver.di-Beschäftigtenbefragung kam zu dem Schluss: „Der Wunsch nach kürzeren Arbeitszeiten erscheint also in erster Linie als ein individueller Gestaltungswunsch und zum Teil ausdrücklich nicht als Wunsch nach kollektiver Arbeitszeitverkürzung" (Lehndorff 2006, S. 178).

Die aktuelle Schwäche der Gewerkschaftsbewegung einerseits und anderer- seits ihre Schwierigkeit, in kollektiven Verträgen individuelle Gestaltungswünsche angemessen umzusetzen, legt vielleicht eine andere Regulierungsinstanz nahe. Dies muss nicht der Staat im engeren Sinne sein. Denkbar wäre etwa auch die Übertragung der Aufgabe an eine Selbstverwaltungseinrichtung von Arbeitgeber- und Arbeitnehmerorganisationen, wie sie die Bundesagentur für Arbeit darstellt. Oder man wagt sich an die Errichtung einer ganz neuen Einrichtung, die - ähnlich wie die Zentralbank die Inflation - dann als Arbeitszeitagentur die Arbeitslosigkeit bekämpft (Grözinger 1998). Diese Idee kann hier nur angedeutet werden. Es wäre lohnenswert, sie zu vertiefen. 
Anger, S. (2006): Zur Vergütung von Überstunden in Deutschland: Unbezahlte Mehrarbeit auf dem Vormarsch, in: DIW-Wochenbericht 15-16, Berlin, S. 189-196

Bauer, F./Groß, H./Lehmann, K./Munz, E. (2004): Arbeitszeit 2003. Arbeitszeitgestaltung, Arbeitsorganisation und Tätigkeitsprofile, Köln Bielinski, H./Bosch, G./Wagner, A. (2002): Wie die Europäer arbeiten wollen: Erwerbs- und Arbeitszeitwünsche in 16 Ländern, Frankfurt/Main u.a.

Böhnke, P./Kohler, U. (2007): Determinanten des Glücks: Lebenszufriedenheit in Europa, in: WSI Mitteilungen 7, S. 373-379

Bosch, G./Schief, S./Schietinger, M. (2005): Trends in der Arbeitszeitpolitik. Zur Diskussion um Dauer und Flexibilisierung der Arbeitszeit sowie der Insolvenzsicherung von Arbeitszeitguthaben, Bonn Buscher, H./Lang, C./Hardt, G. (2005): The performance of European labour markets on the basis of data obtained from the June 2004 ad hoc labour market survey. Final report of the Institut für Wirtschaftsforschung Halle, Halle (Saale)

Fischer, L., (Hrsg.) (2006): Arbeitszufriedenheit: Konzepte und empirische Befunde, Göttingen

Franz, W. (2004): Winds of Change? - Von der Verkürzung zur Verlängerung der Wochenarbeitszeit, in: Zeitschrift für Wirtschaftspolitik 3, S. 325332

Franz, W. (2006): Arbeitsmarktökonomik, Berlin

Frey, B. S./Stutzer, A. (2002): Happiness and economics : how the economy and institutions affect well-being, Princeton u.a.

Grözinger, G. (1998): Drei wirtschaftspolitische Ziele, drei semi-autonome Institutionen, in: Heise, A. (Hrsg.): Renaissance der Makroökonomik, Marburg, S.141-178

Grözinger, G./Matiaske, W. (2004): Regional unemployment and individual satisfaction, in: Grözinger, G./van Aaken, A. (Hrsg.): Inequality: New Analytical approaches, Marburg, S.87-104

Holst, E. (2007): Arbeitszeitwünsche von Frauen und Männern liegen näher beieinander als tatsächliche Arbeitszeiten, in: DIW-Wochenbericht 14-15, S. 209-215

Holst, E./Schupp, J. (1998): Arbeitszeitpräferenzen in West- und Ostdeutschland 1997. Potential für Verkürzung der Arbeitszeit gesunken, in: DIW-Wochenbericht 37, S. 667-677

Holst, E./Schupp, J. (2000): Förderung von Teilzeitarbeit durch gesetzlichen Rechtsanspruch - Reform oder Hindernis für mehr Beschäftigung?, in: DIW-Wochenbericht 49, S. 829-837

Holst, E./Trzcinski, E. (2005): Geburt eines Kindes erhöht die Lebenszufriedenheit der Mütter nur kurzfristig: geringe Lebenszufriedenheit insbesondere bei türkischen Müttern, in: DIW-Wochenbericht 74, S. 69-76 Kahneman, D./Diener, E./Schwarz, N. (2003): Well-being: The foundations of hedonic psychology, New York
Layard, P. R. G. (2005): Happiness: lessons from a new science, London Lehndorff, S. (2001): Weniger ist mehr. Arbeitszeitverkürzung als Gesellschaftspolitik, Hamburg

Lehndorff, S. (2006): Sicherheit anbieten, Vielfalt ermöglichen. Über Krise und Reformen der Arbeitszeitregulierung, in: Lehndorff, S. (Hrsg.): Das Politische in der Arbeitspolitik. Ansatzpunkte für eine nachhaltige Arbeitszeit- und Arbeitszeitgestaltung, Berlin, S.157-194

Matiaske, W./Mellewigt, T. (2001): Arbeitszufriedenheit: Quo vadis?, in: Die Betriebswirtschaft 1, S. 7-24

McKinsey and Company (1994): Teilen und Gewinnen. Das Potential der flexiblen Arbeitszeitverkürzung, München

Merz, J. (2002): Time and economic well-being - a panel analysis of desired versus actual working hours, in: Review of Income and Wealth 3, S. 317-346

Mischau, A./Oechsle, M. (Hrsg.) (2005): Arbeitszeit - Familienzeit - Lebenszeit: Verlieren wir die Balance? Zeitschrift für Familienforschung. Sonderheft 5, Wiesbaden

Nachreiner, F./Rädiker, B./Janßen, D./Schomann, C. (2005): Untersuchungen zum Zusammenhang zwischen der Dauer der Arbeitszeit und gesundheitlichen Beeinträchtigungen, Oldenburg

Rürup, B./Gruescu, S. (2005): Familienorientierte Arbeitszeitmuster. Neue Wege zu Wachstum und Beschäftigung, Berlin

Schief, S. (2003): Arbeitszeiten in Deutschland: eine Frage der Messung?, in: Mitteilungen aus der Arbeitsmarkt- und Berufsforschung 2, S. 187198

Schildt, G. (2006): Das Sinken des Arbeitsvolumens im Industriezeitalter, in: Geschichte und Gesellschaft 1, S. 119-148

Schwarze, J./Andersen, H. H./Anger, S. (2000): Self-rated Health And Changes In Self-rated Health As Predictors Of Mortality - First Evidence From German Panel Data, Berlin

Seifert, H. (1993): Ausmaß und Effekte der Arbeitszeitverkürzung, in: Hampe, P. (Hrsg.): Zwischenbilanz der Arbeitszeitverkürzung, München, S.11-28

Spitznagel, E./Wanger, S. (2004): Mehr Beschäftigung durch längere Arbeitszeiten? Ein Beitrag zu der Diskussion um eine generelle Erhöhung der Arbeitszeit, Nürnberg

Spitznagel, E./Wanger, S./Zika, G. (2005): Arbeitszeitpolitik, in: Allmendinger, J.,/Eichhorst, W. /Walwei, U. (Hrsg.): IAB Handbuch Arbeitsmarkt. Analysen, Daten, Fakten, Frankfurt/M., S.143 - 192

Trzcinski, E./Holst, E. (2003): Hohe Lebenszufriedenheit teilzeitbeschäftigter Mütter, in: DIW-Wochenbericht 35, S. 539-549

Wagner, G. G./Frick, J. R./Schupp, J. (2006): Enhancing the Power of Household Panel Studies - The Case of the German Socio-Economic Panel Study (SOEP), DIW Data Documentation 2006-13, Berlin 